\title{
TRAUMATISMOS DENTOALVEOLARES, CARACTERÍSTICAS CLÍNICAS E IMAGENOLÓGICAS: UNA REVISIÓN DE LA LITERATURA
}

\author{
DENTOALVEOLAR TRAUMATISM, CLINICAL AND IMAGENOLOGICAL \\ CHARACTERISTICS: A LITERATURE REVIEW
}

Mónica Pamela Sánchez Herrera

\section{RESUMEN}

El traumatismo dentoalveolar resulta del impacto de una fuerza externa, con variación de intensidad, que afecta la calidad de vida del individuo debido a la alteración en la estética por alteración de la apariencia, problemas funcionales al alterar el habla, lo que genera un impacto psicológico y social. Las causas van desde una simple caída hasta prácticas deportivas, accidentes automovilísticos, violencia, manejo de herramientas de trabajo. Los niños y adolescentes son los más susceptibles, y las piezas anteriores superiores las que frecuentemente son más afectadas. El manejo multidisciplinario de la lesión y el estudio con exámenes complementarios, como la radiografía, son indispensables para el diagnóstico de los traumatismos. Asimismo, avances tecnológicos como la tomografía de haz cónico han mejorado la observación al proporcionar cortes transversales.
Palabras clave: traumatismos de los dientes, tomografía computarizada de haz cónico, resorción radicular, anquilosis

\section{ABSTRACT}

Dentoalveolar trauma results from the impact of an external force, of varying intensity, affecting the quality of life of the individual due to aesthetic alteration caused by an altered appearance, and functional problems caused by alteration of speech, producing a psychological and social impact. Causes may range from a simple fall, to sporting activities, car accidents, violence, or the use of tools in the workplace. Children and adolescents are the most susceptible groups and the anterior superior areas are those most often affected. Multidisciplinary management of the injury and studies involving complementary tests such as radiography are essential for the diagnosis of trauma. Technological advances such as

Citar como: Sánchez-Herrera MP. Traumatismos dentoalveolares, características clínicas e imagenológicas: una revisión de la literatura. Rev Cient Odontol (Lima). 2018; 6 (2): 195-212.

${ }^{1}$ Universidad Mayor de San Andrés (La Paz, Bolivia). 
the use of cone beam tomography have improved diagnosis by providing cross section scans.

Keywords: Tooth injuries, cone beam computed tomography, root resorption, ankylosis

\section{INTRODUCCIÓN}

El trauma se describe como una lesión resultante de una fuerza externa, con patrón de intensidad impredecible, y es considerado un problema social, pues representa un riesgo a nivel mundial (1-3). En la actualidad, el trauma dental tiene una alta prevalencia en niños y adolescentes, y se ha convertido en un problema de salud pública que afecta la calidad de vida, causa problemas estéticos y funcionales, y genera un impacto psicológico y social al afectar la apariencia, el habla y la interacción social de los pacientes (1, 3-7). La mayoría de los traumatismos y desplazamientos son resultados de caídas, actividades deportivas, accidentes de tránsito, accidentes laborales que involucran tensión física por el uso de herramientas, violencia comunitaria y violencia doméstica. De acuerdo con diferentes estudios, la prevalencia es mayor en varones a excepción de la violencia doméstica, en la que la prevalencia son mujeres $(1,3,8,9)$.

En los tres primeros años de vida, ocurren muchos accidentes que afectan el niño porque aprende a inclinarse, gatear, caminar, pararse, y pasa de una dependencia total de movimientos a una estabilidad propia. Las caídas consecuentes causan lesiones faciales y traumatismos que representan un desafío en su manejo y diagnóstico para los profesionales de la salud, que deben evitar un peligro en el patrón de crecimiento del esqueleto facial, por los posibles cambios morfológicos y anatómicos. En la mayoría de los casos, el trata- miento inmediato y adecuado minimiza la angustia emocional de los pacientes y familiares (10-12).

Las lesiones traumáticas de los dientes y las estructuras de soporte son una de las pocas áreas de la odontología consideradas como una emergencia, donde la tarea principal del odontólogo es garantizar la viabilidad de los dientes traumatizados, motivo por el cual el manejo multidisciplinario con diferentes especialistas es muy importante (12-15). El pronóstico del traumatismo está influido por el grado, el tipo de trauma y la madurez del diente (6). Las lesiones pueden ser resultado de un trauma directo o indirecto, de los cuales el primero es más frecuente en el maxilar debido a la exposición de los dientes anteriores. La complejidad y la naturaleza de los traumatismos dentoalveolares varía considerablemente, ya que puede sufrir 108 condiciones distintas de trauma, que involucran los dientes y sus estructuras de soporte (12-15). La frecuencia de extrusión total o avulsión aumenta con la edad, mientras que la lesión por intrusión disminuye con la edad. Una pieza dentaria traumatizada o fragmento puede desplazarse hacia adelante, atrás o lateralmente de acuerdo con la energía de impacto y dirección del agente causal, además de la ubicación de la lesión y la estructura de soporte de la pieza afectada (12-14). Las lesiones de trauma dental son de naturaleza iatrogénica cuando ocurren durante la intubación de anestesia general, también para colocación de piercings en la lengua $(16,17)$ o causados por el profesional durante el acto quirúrgico de la extracción de una pieza dentaria, por la fuerza excesiva aplicada al momento de realizar la cirugía (18).

El propósito del presente trabajo es realizar la revisión de literatura de las características clínicas e imagenológicas de los traumatismos dentoalveolares, así como de los factores de riesgo y el 
manejo de los pacientes traumatizados, para facilitar al profesional el desarrollo de sus capacidades frente a este tipo de situaciones.

\section{CAUSAS DE TRAUMATISMOS Y FACTORES DE RIESGO}

El trauma en las piezas dentarias anteriores superiores y sus tejidos de soporte es relativamente común entre niños y adolescentes (19). En el primer caso, se debe a que, al aprender a caminar, por su débil equilibrio, los niños tienden a caerse y lesionarse con mayor frecuencia la porción coronaria de las piezas dentarias, y en el segundo caso, puede atribuirse a la realización de deportes de contacto o de alto riesgo, violencia, accidentes de tránsito, caídas o accidentes médicos dentales.

Otros factores considerados de riesgo para la lesión traumática son la maloclusión clase II, la mordida abierta y la mordida cruzada, el aumento de overjet y la inadecuada cobertura labial de los incisivos superiores. Estos factores afectan la dentición permanente porque implican la pérdida de estructura dental extensa por un mayor porcentaje de luxaciones, avulsiones e intrusiones, o una combinación con fractura radicular. Los niños son más afectados que las niñas y la edad de incidencia de trauma fluctúa entre los 6 y los 15 años. Por este motivo, es muy importante que reciban el tratamiento lo antes posible, a fin de prevenir y evitar complicaciones relacionadas con la erupción dental, el desarrollo alveolar, la oclusión y el crecimiento facial $(1,5$, $10,13,16,17,20-27)$. Además del dolor y la incomodidad de la lesión, la alteración en la apariencia del niño puede convertirlo en objeto de burla por parte de otros niños, por las posibles deformidades que puede causar el traumatismo $(27,28)$.
El traumatismo dentoalveolar en adultos suele ocurrir por actividades deportivas, violencia, accidentes por consumo de alcohol, drogas, accidentes de tránsito, asaltos, accidentes laborales, entre otros, siendo la mayoría resultado de un incidente único $(3,6-9,13,22,29-35)$.

En pacientes con habilidades especiales o que padecen trastornos cerebrales como epilepsia, las complicaciones dentofaciales son generalmente la intrusión y la avulsión dental $(27,36)$. Algunas lesiones por trauma dental pueden ocurrir debido a iatrogénica, como en el caso de accidentes durante la intubación para colocar anestesia general o por la colocación de piercings en la lengua, que causan fracturas coronarias o radiculares, lesiones por luxación o avulsión (16, 17). Así mismo, pueden presentarse traumatismos causados por profesionales durante el acto quirúrgico, por ejemplo, la exodoncia de una pieza dentaria, por la relación con estructuras anatómicas próximas, debido a la aplicación de fuerzas excesivas durante el tratamiento o por presencia de variaciones anatómicas 0 patológicas. Generalmente, se presenta adelgazamiento de corticales, raíces divergentes, anomalías dentales, anquilosis, hipercementosis y lesión periapical con esclerosis ósea (18) (figura 1).

La gravedad de las lesiones varía de acuerdo con el tipo, ubicación, dirección, energía del impacto, elasticidad y forma del objeto impactante, así como la resistencia y reacción de los tejidos que rodean el área afectada. La lesión puede provocar desplazamiento, rotación e intrusión, siendo las fracturas coronarias las más comunes, seguidas por las luxaciones dentales, debido a la mayor resiliencia del tejido óseo. En pacientes jóvenes, los traumatismos por concusión y subluxación son los de mayor prevalencia, sobre todo en varones $(4,16,17$, $24,29,35)$. 

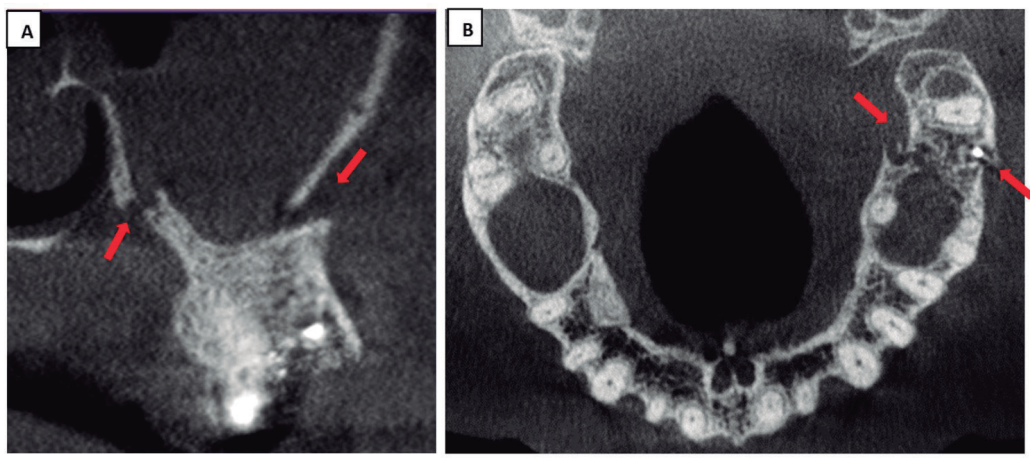

FIGURA 1. COMPLICACIÓN POSTERIOR A EXODONCIA CON FRACTURA DE CORTICALES VESTIBULAR Y PALATINO, CON DESPLAZAMIENTO HACIA VESTIBULAR Y COMUNICACIÓN BUCO SINUSAL, REALIZADO CON TCHC. A. VISTA TRANSVERSAL. B. VISTA AXIAL.

Las piezas dentarias que tienen mayor prevalencia de sufrir traumatismos son los incisivos maxilares centrales (25, $37,38)$ y laterales; los caninos rara vez están involucrados (14). Esta alta prevalencia se debe a que podrían tener posición o proyección expuesta en el arco dental o podría tratarse de una mayor posición protusiva, tamaño, además de la falta de protección del labio (labio corto) $(4,5,10,17,24)$. Las lesiones traumáticas pueden variar desde pequeñas líneas de esmalte hasta la avulsión del diente (40). La fractura de la corona es la lesión más frecuente de la dentición permanente y representa un peligro para la pulpa dental (39). Las fracturas coronales en piezas dentales anteriores superiores crean problemas estéticos al paciente, por ello se requiere una reparación rápida por las necesidades estéticas y funcionales (37). La importancia de la planificación del tratamiento con enfoque multidisciplinario para el manejo óptimo de las lesiones traumáticas incluye diferentes especialidades como cirugía maxilofacial, radiología, odontopediatría, endodoncia, periodoncia, rehabilitación protésica, ortodoncia y, sobre todo, estética, por el impacto en la psicología del paciente $(16,24,26,36$, 37, 39-43) El cirujano maxilofacial o el odontopediatra, por lo regular, es quien brinda la atención primaria de urgencia (15). La ubicación de la línea de frac- tura y el estado periodontal son factores importantes para la conducta a seguir en el tratamiento y conservación, en lo posible, del diente traumatizado y del área adyacente a la lesión $(44,45)$, así como la colaboración entre profesionales médicos y odontológos (17). Es necesaria la combinación de procedimientos de diferentes especialidades para la funcionalidad y restauración de la pieza (42).

\section{MANEJO DEL PACIENTE TRAUMATIZADO}

El tratamiento del trauma dental no es una situación común en la práctica diaria, es un desafío por la rareza de la situación las diferentes posibilidades de diagnóstico, que involucra los dientes y los tejidos de soporte $(12,16)$. El tratamiento va a estar relacionado con el conocimiento y habilidades del profesional; sin embargo, la baja contribución de incidencia de traumatismos dentoalveolares en la práctica clínica genera un bajo conocimiento y manejo de las lesiones para un odontólogo general. El acceso a la información por parte de los profesionales jóvenes los prepara mejor para el manejo de un traumatismo dentoalveolar $(12,14,35$, 46, 47). La evaluación inicial correcta y el tratamiento adecuado, además del pronóstico, dependerán del tiempo 
transcurrido desde el incidente, del examen minucioso, aliviar al paciente del dolor y controlar la hemorragia, evaluación de la dentición, la posibilidad de aspiración de dientes o fragmentos de dientes, el análisis radiológico y la monitorización. Si por la distancia o la hora del accidente no es posible ver inmediatamente al especialista, con la tecnología es posible realizar una teleconsulta $(6$, $10,29,48)$.

El empleo de exámenes complementarios, como la radiografía, es esencial para establecer el diagnóstico y descartar un posible traumatismo (44, 49), porque proporciona información de la etapa de formación radicular. Si las lesiones afectan tejidos duros dentales, el ligamento periodontal y el hueso alveolar (12). La radiografía intraoral se recomienda para la detección inicial del traumatismo, no solo para el diagnóstico preciso, sino para establecer la comparación posterior al tratamiento, se sugiere realizar varias tomas en radiografías periapicales, de preferencia con la técnica de cono largo debido a su estandarización (50) en diferentes ángulos. Así mismo, una radiografía oclusal para observar fragmento incrustados en tejido blando como en labios (17) y una radiografía panorámica en traumatismos extensos; sin embargo, por la limitación de las películas planas respecto de la proyección y la superposición de imagen de las estructuras dentales, no proporciona una imagen con definición clara para el diagnóstico de fracturas alveolares, desplazamiento dental y fractura radicular $(6,11,13,26,49,51)$.

La tomografía computarizada de haz cónico $(\mathrm{TCHC})$, primordial para determinar el tipo de traumatismo (17), ha mejorado la precisión de los diagnósticos de lesiones traumáticas $(25,30)$, pues proporciona un alto nivel de detalle en poco tiempo y permite la generación de imágenes tridimensionales (51), aunque no ha sustituido el uso de imágenes planas. Es recomendada para observar detalles y en sospecha de fracturas radiculares y alveolares $(6,49,52)$, y proporciona una radiación menor en comparación con la tomografía médica convencional (52). Para los cirujanos maxilofaciales, es importante el acceso a imágenes transversales y la capacidad de evaluar corticales vestibular, lingual o palatina, ha superado las limitaciones de la superposición de estructuras, magnificación y distorsión $(14,25,40,49)$, y proporciona una excelente visualización tridimensional de las reabsorciones radiculares durante los exámenes de control (6).

\section{CORRELACIÓN CLÍNICA E IMAGENOLÓGICA}

Al producirse el traumatismo dentoalveolar, la línea de la fractura puede extenderse en diferentes direcciones, lo que determina el plan de tratamiento (40). Para el diagnóstico de los diferentes tipos de traumatismos, debe existir una interrelación clínica y radiológica (10). El sistema más utilizado es el sistema WHO-Andreasen, de 1992, útil para clasificar las lesiones en los tejidos duros dentales y la pulpa, así como en el periodonto (44).

\section{CLASIFICACIÓN DE LAS LESIONES DENTALES}

Las lesiones dentales traumáticas se clasifican de la siguiente manera: (i) fisura del esmalte, (ii) fractura del esmalte, (iii) fractura del esmalte-dentina sin exposición de la pulpa, (iv) fractura del esmaltedentina con la exposición de la pulpa, (v) fractura vertical coronaria, (vi) fractura radicular, (vii) concusión, (viii) subluxación, (ix) luxación lateral, (x) intrusión y (xi) avulsión. Los tipos i-iv se clasifican como lesiones de tejidos duros; v-vi, como fracturas de raíz, y vii-xi, como 
lesiones periodontales (5). El trauma dental se clasifica en lesiones de tejido duro (fractura de esmalte y astillado del esmalte, fractura de esmalte-dentina sin afectación pulpar, fractura de esmaltedentina con compromiso pulpar, fractura de raíz, fractura de raíz de cuervo y lesiones periodontales), concusión, subluxación, intrusión, extrusión, luxación lateral y avulsión (53).

La fractura coronaria puede ser de tipo vertical, horizontal u oblicua, y comprometer o no la pulpa dentaria. Si fuera el caso, se debe realizar un tratamiento de endodoncia de acuerdo con la sintomatología clínica e imagenológica, y la rehabilitación estética. De no estar comprometido el tejido pulpar, solo se debe realizar la estética, verificando el límite de la fractura, si existe alguna complicación, a nivel apical se observará con radiografía periapical $(39,54)$. La línea de fractura coronaria localizada a nivel subgingival representa un desafío para el tratamiento, por el difícil procedimiento que lleva en tratamientos de endodoncia y de restauración $(37,44)$.

Las fracturas compuestas corono-radiculares involucran esmalte, dentina, pulpa y cemento. Radiográficamente, se observa que la línea de fractura se encuentra por debajo del límite subgingival, a nivel de la cresta del hueso alveolar interdental (20). El manejo de este tipo de traumatismos compuestos es un desafío, ya que la fractura requiere el reposicionamiento del margen de fractura supragingival para la ferulización sobre tejido sano y su posterior restauración (42) (fig. 2). La fractura radicular involucra cemento, dentina, pulpa y periodonto, y puede ser de tipo vertical, horizontal u oblicua, siendo las dos últimas las más comunes, y pueden ser múltiples o únicas (38). En la fractura de tipo vertical radicular puede existir movilidad dentaria, para lo cual se solicita una radiografía periapical, de la cual se necesitan varios planos para identificar o descartar la lesión, y la tomografía de haz cónico TCHC, para evaluar la lesión, ya que muestra cortes transversales la lesión de la o las piezas dentarias y de las corticales óseas que pueden estar involucradas. Un protocolo de tratamiento de más de 30 años para fractura radicular ha sido la ferulización rígida por dos a tres meses, con controles radiográficos hasta los dos años $(10,26$, 38, 40) (fig. 3).

La fractura radicular horizontal es frecuente a nivel del tercio medio radicular, tercio apical radicular y tercio coronal, según la gravedad de la lesión (39). Presenta dolor severo prolongado al impulso eléctrico, térmico, percusión
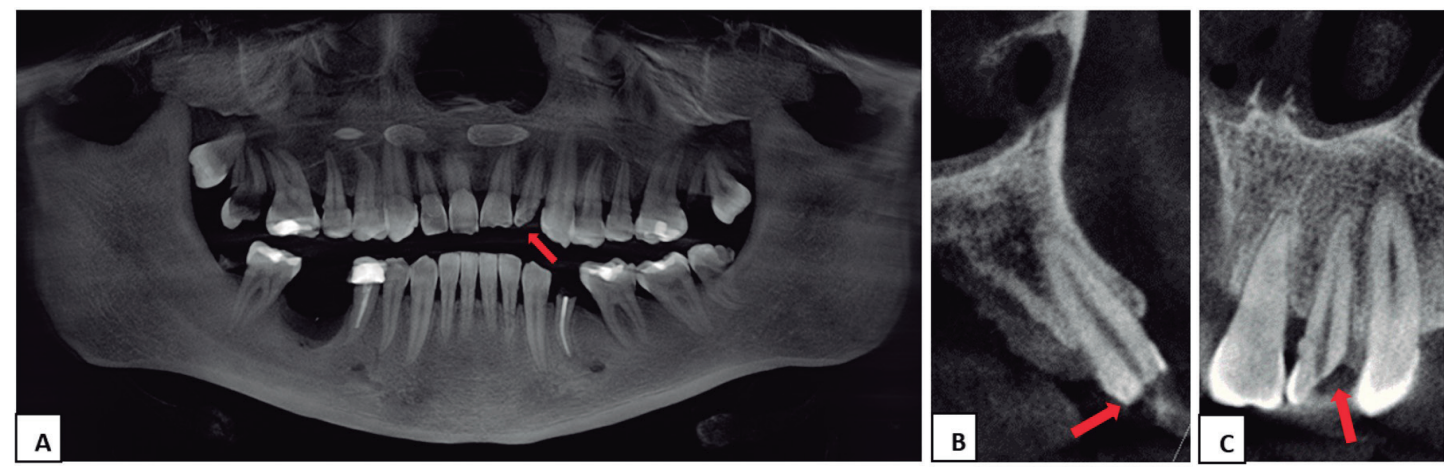

Figura 2. Fractura coronaria-radicular en TCHC. A. Vista panorámica. B. Vista transversal. C. VISTA TANGENCIAL. 

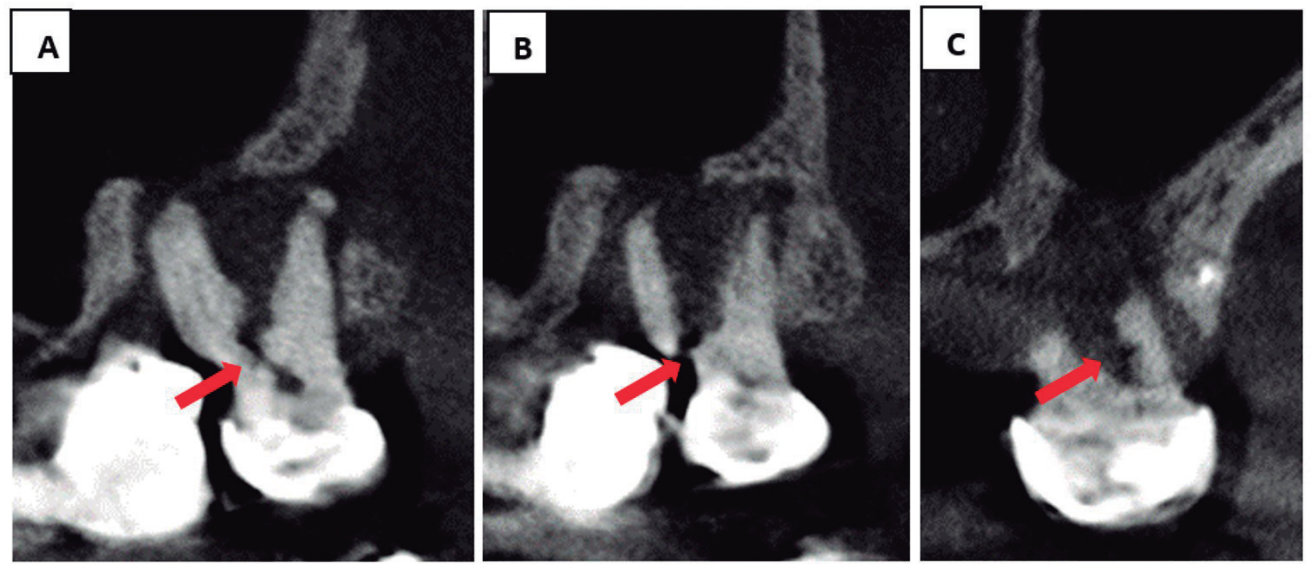

Figura 3. Fractura Vertical RADICULAR EN TCHC. A. Vista tangenCIAL, SE ObSERVA Fractura VERTICAL A NIVEL DE FURCA. B. VISTA TANGENCIAL FRACTURA VERTICAL A NIVEL DEL TERCIO CERVICAL

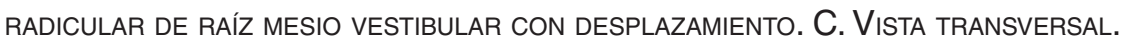
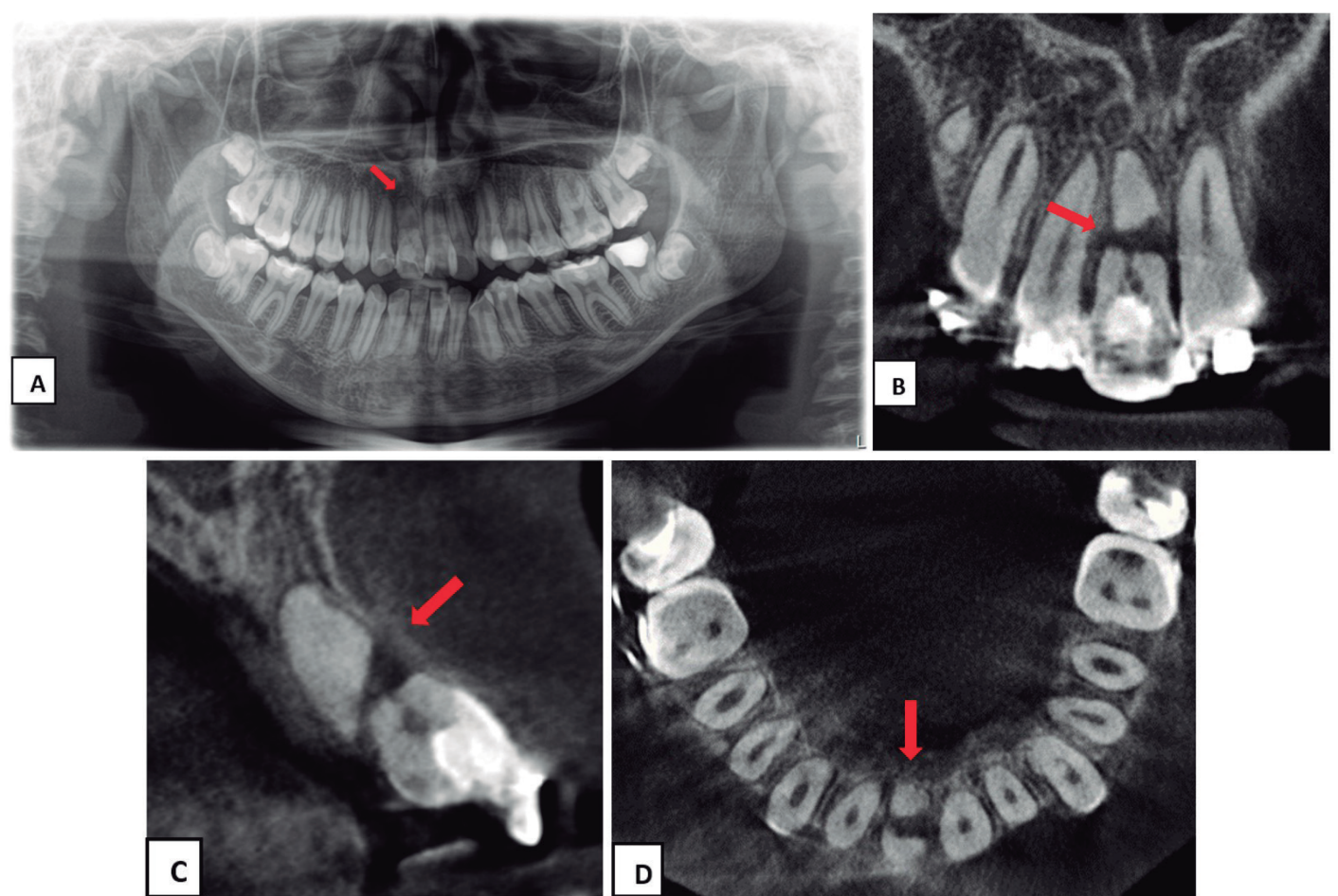

Figura 4. Fractura horizontal a nivel tercio medio radicular con desplazamiento. A. Radiografía PANORÁmicA. B. TCHC VISTA TANGENCIAL. C. TCHC VISTA TRANSVERSAL. D. TCHC VISTA AXIAL.

vertical y palpación. Radiográficamente, puede estar la pieza completamente desarrollada o no depende de la edad del desarrollo dental del paciente, se observará una línea horizontal con o sin desplazamiento del segmento coronal. Los controles con radiografía se pueden realizar para observar la formación de tejido duro entre los fragmentos y clínicamente si la pieza dentaria es estable y funcional (55) (fig. 4).

En la fractura radicular oblicua, mediante una radiográfica periapical con diferentes angulaciones, se puede observar el recorrido y extensión del trazo de fractura 

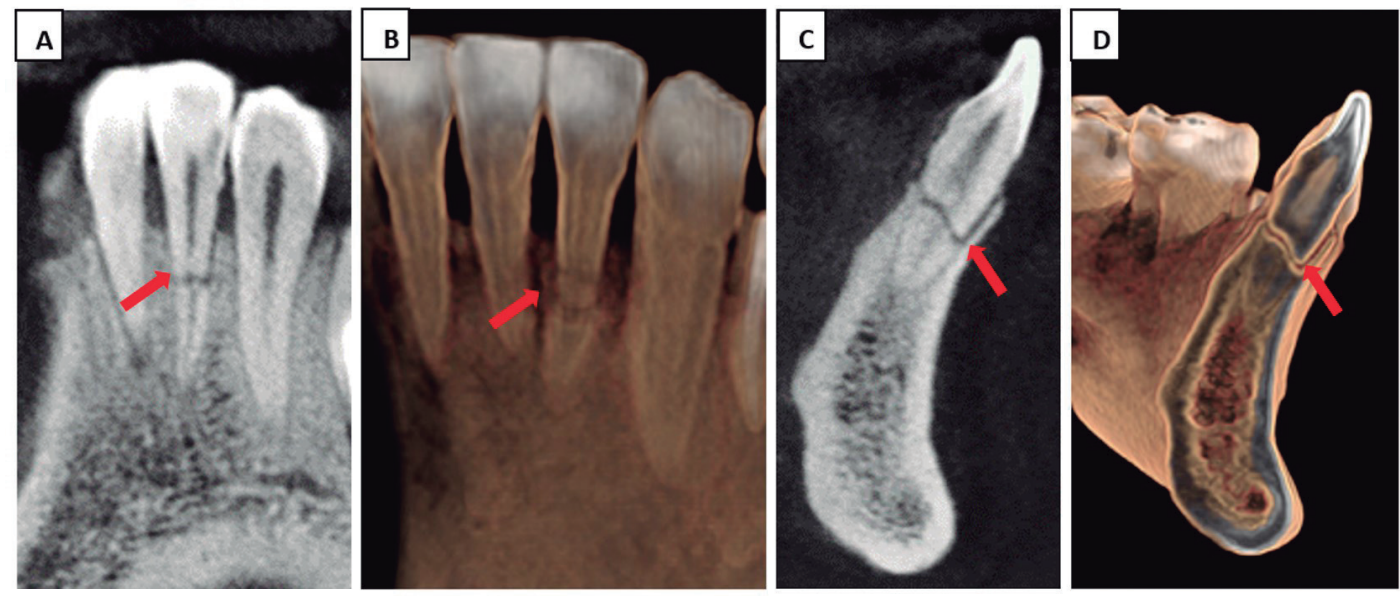

FIGURA 5. FRACTURA OBLICUA NIVEL TERCIO MEDIO RADICULAR CON DESPLAZAMIENTO REALIZADO CON TCHC. A. VISTA TANGENCIAL B. VISTA TANGENCIAL EN RECONSTRUCCIÓN RENDERIZADA C. Vista tRANSVERSAL D. VISTA TRANSVERSAL EN RECONSTRUCCIÓN RENDERIZADA.
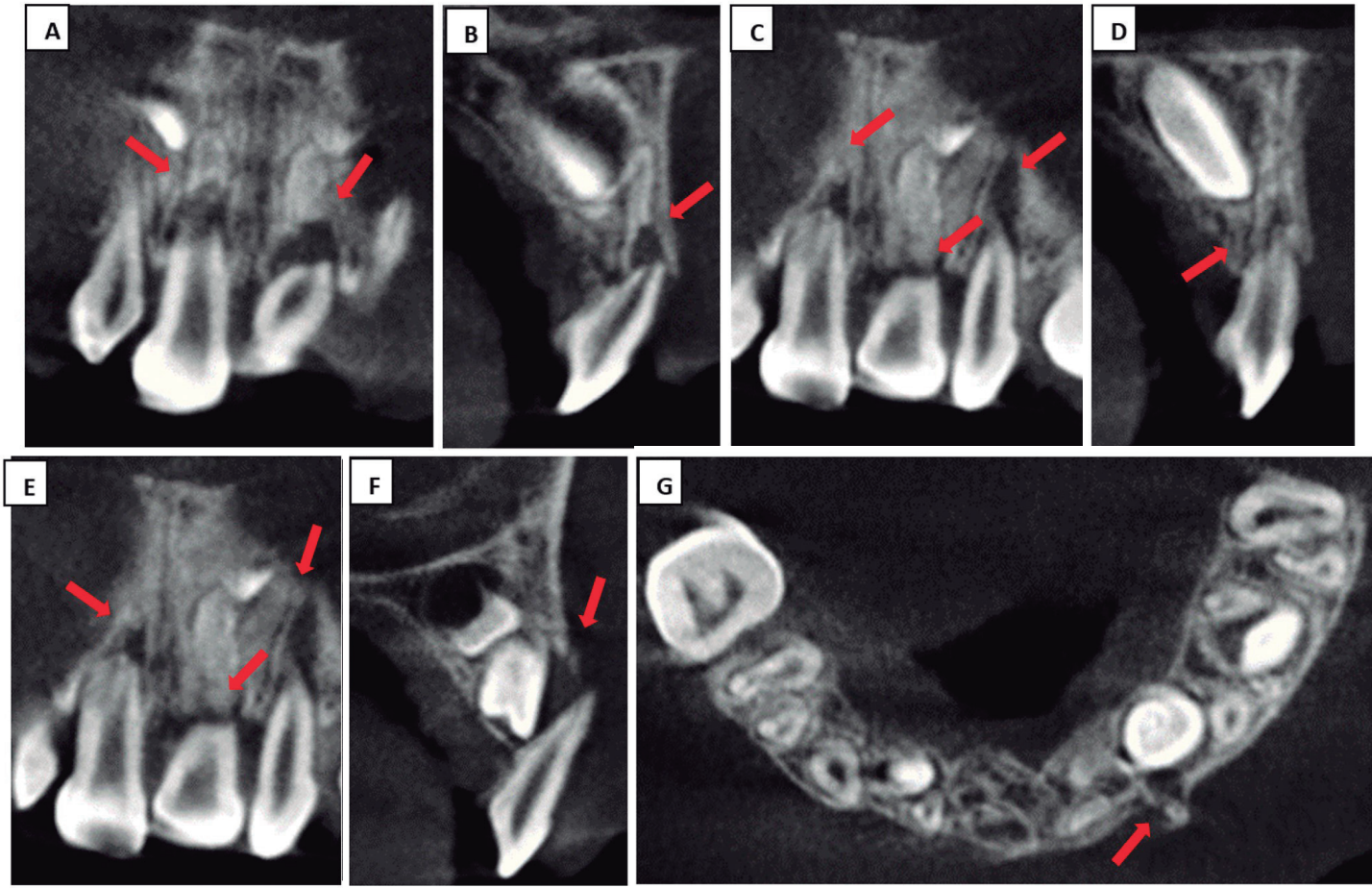

FIGURA 6. LUXACIÓN MÚLTIPLE EN PIEZAS ANTERO SUPERIORES TEMPORARIAS CON DESPLAZAMIENTO HACIA PALATINO, SE OBSERVA AUMENTO DEL ESPACIO DEL LIGAMENTO PERIODONTAL Y RUPTURA DE CORTICAL Vestibular Y PALATina REALizAda CON TCHC. A, C, E. Vista tangencial B, D, F. Vista transversal. G. VISTA AXIAL.

a lo largo de los tres tercios radiculares, si existe exposición pulpar, la ubicación de la fractura por mesial o distal. Controles cada 6 meses muestran la extensión de la extrusión y la obturación radicular luego del tratamiento multidisciplinario $(38,45)$.
Controles radiográficos en periodos de 3 meses permiten evaluar la posibilidad de reabsorción radicular (42) (fig. 5).

El tipo de traumatismo dentoalveolar que afecta a los tejidos periodontales más común son las luxaciones, en un 
$15 \%$ a un $61 \%$ (24). Clínica y radiográficamente, las piezas luxadas tienen las coronas desplazadas hacia el paladar, que provocan la conminución o fractura del hueso alveolar labial y causar lesión a los ligamentos periodontales (fig. 6). Se debe descartar radiográficamente que existan más lesiones o piezas dentarias comprometidas, como grietas o fracturas de esmalte con radiografía periapical y radiografía lateral. Luego del reposicionamiento se toma una radiografía periapical para su evaluación, y controles a los 2 años muestran demanda estéticas y funcionales satisfactorias (24).

La subluxación es un tipo de trauma menos complejo, favorable para los tejidos lesionados, que causa un aumento de la movilidad dentaria en sentido horizontal y sensibilidad a la percusión y oclusión. En general, radiográficamente no muestra alteración de estructuras; sin embargo, en el caso de movilidad aguda se puede observar un engrosamiento del espacio del ligamento periodontal, pero es necesario realizar controles por la posibilidad de necrosis pulpar (4). En una TCHC se puede observar fractura con desplazamiento $\mathrm{y}$, además, otras estructuras que pudieran estar comprometidas (52) (fig. 7).

La concusión es una lesión de las estructuras de soporte de la pieza dentaria que sufren traumas menores, con mínima movilidad dental o desplazamiento. Clínicamente, existe reacción a la percusión vertical u horizontal, con o sin asociación de fractura coronaria; no es evidente un cambio en estructuras en la radiografía. Realizar controles radiográficos es importante por la posibilidad de reabsorción radicular $(4,12)$.

La intrusión es el desplazamiento axial de la pieza dentaria hacia el hueso alveolar, de buen pronóstico $(23,25)$,
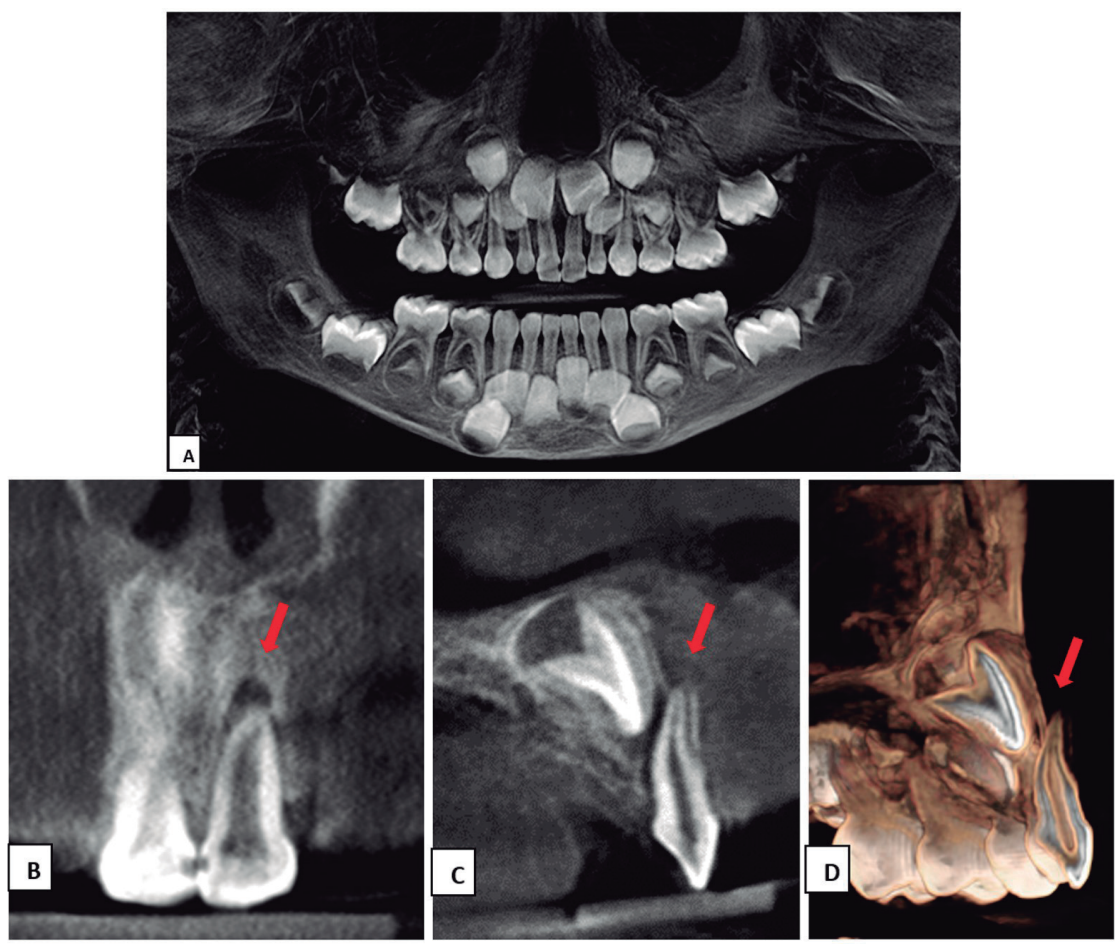

FIGURA 7. LUXACIÓN LATERAL EN PIEZA ANTERO SUPERIOR TEMPORARIA CON DESPLAZAMIENTO, SE OBSERVA AUMENTO DEL ESPACIO DEL LIGAMENTO PERIODONTAL Y RUPTURA DE CORTICAL VESTIBULAR REALIZADO CON TCHC. A. Vista panorámica. B. Vista tangencial. C. Vista transversal. D. Vista transversal en RECONSTRUCCIÓN RENDERIZADA. 

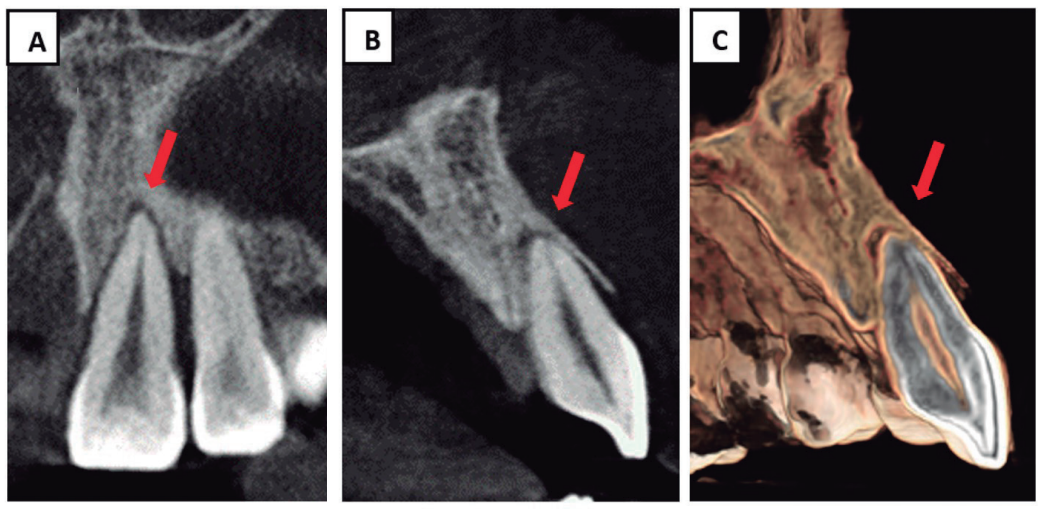

FIGURA 8. EXTRUSIÓN DENTARIA EN PIEZA 2.1 CON LIGERO DESPLAZAMIENTO HACIA PALATINO, SE OBSERVA AUMENTO DEL ESPACIO DEL LIGAMENTO PERIODONTAL EN TODO EL RECORRIDO ALVEOLAR, REALIZADO CON TCHC. A. Vista tangencial. B. Vista transversal. C. Vista transversal en ReConstrucción RENDERIZADA.
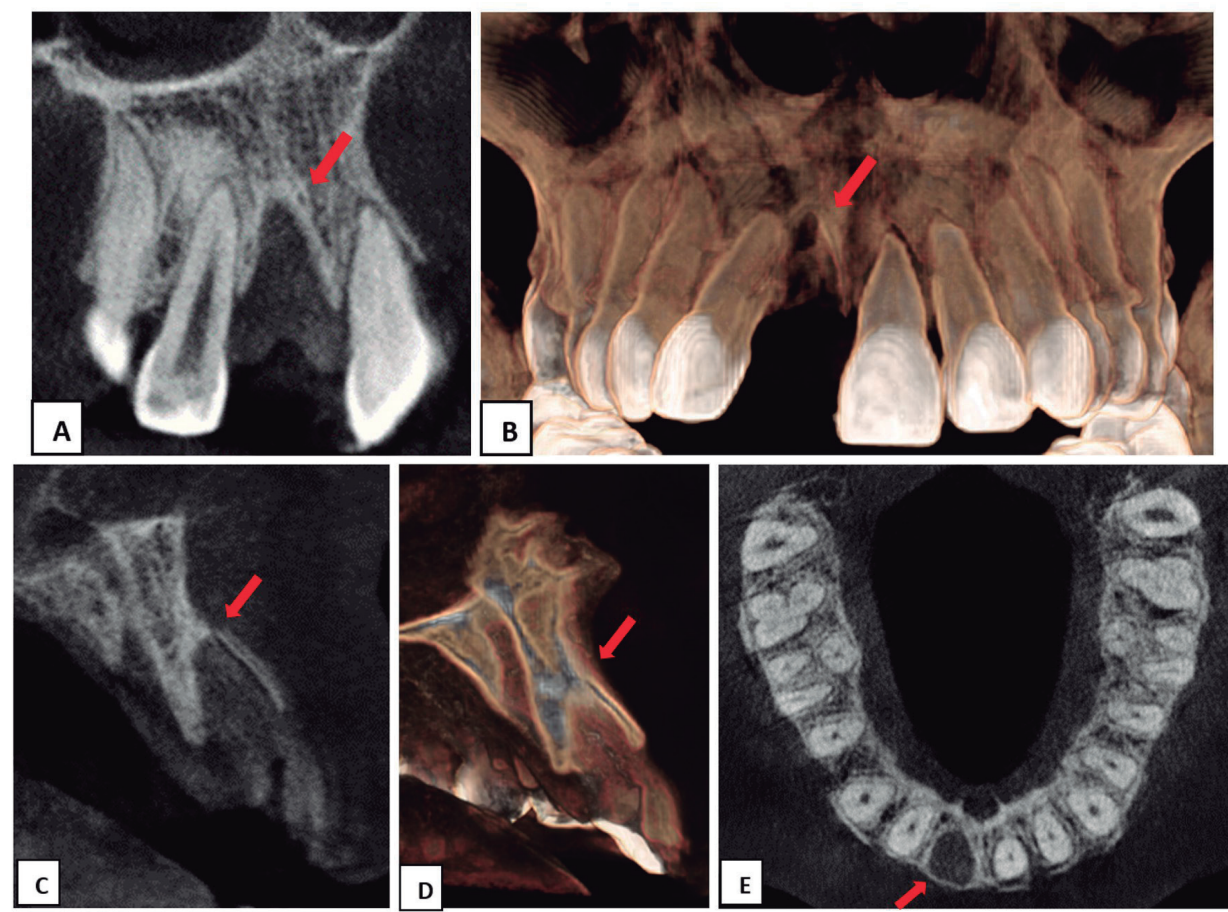

Figura 9. AvULSIÓN DENTARIA EN PIEZA 1.1, SE OBSERVA LECHO ALVEOLAR CON LÁMINA DURA, RUPTURA DE A CORTICAL Vestibular, REALIZADO CON TCHC. A. Vista tangencial. B. Vista RENDERIZada.

C. Vista tRansversal. D. Vista transVersal en ReCONStrucción Renderizada. E. Vista axial.

que generalmente produce fractura a nivel alveolar (56). Es el resultado del impacto directo en el borde incisal en una dirección axial y la energía de impacto puede fracturar la corona, cuya consecuencia posterior es la anquilosis o reabsorción radicular, pérdida ósea marginal, que se puede observar radiográficamente en panorámica o periapical, además de estar por encima del plano de oclusión, ensanchamiento del espacio del ligamento periodontal, controles con radiografía periapical luego del tratamiento de endodoncia y extrusión con ortodoncia, sea inmediata o tardía (23, $26,27,56,57)$. El diagnóstico se complementa mejor con TCHC (25), controles con radiografía panorámica $(25,28)$. 
La extrusión dentaria o avulsión parcial es el desplazamiento axial parcial hacia fuera del alveolo. Clínicamente, el diente parece alargado, generalmente desplazado hacia el palatino y con movilidad dentaria excesiva (58). Radiográficamente, se observa en radiografía periapical un ensanchamiento mayor del espacio del ligamento periodontal, desplazamiento coronal de las piezas dentarias que no ha sido expuesto a la desarticulación dentoalveolar en la $\mathrm{TCHC}$, se observa la posibilidad de fractura de cortical vestibular, la cual como control se debe volver a tomar una $\mathrm{TCHC}$ $(29,58,59)$ (fig. 8).

La avulsión o extrarticulación es el traumatismo dentoalveolar más frecuente. Es el desplazamiento completo de la pieza dentaria fuera del alveolo, ocasionado por un trauma con alta resiliencia de la estructura de soporte del diente por el desgarro de las fibras del ligamento periodontal $(19,27,60,61)$. Se puede evaluar con una radiografía panorámica, además de la avulsión si existe fractura dentoalveolar de otras piezas dentarias (1) (fig. 9). Así mismo, verificar la posibilidad de fragmentos enterrados en mucosa, que pueden ser observados con una radiografía oclusal, además tomar una radiografía del tórax, ya que los fragmentos o la pieza entera son inhaladas y generalmente son vistas en los bronquios, principalmente el derecho. Una TCHC permite evaluar la oclusión del paciente, la movilidad de dientes y alveolos $(1,61)$. La reimplantación de la pieza dentaria produce el mejor pronóstico, siempre y cuando la pieza se haya sometido a un proceso de almacenamiento apropiado y en los primeros 5 minutos (60-64), al ser reimplantado y luego ferulizado, realizar un control con radiografía para valorar la posición de la pieza (6). Controles posteriores para observar la reabsorción interna y externa, o anquilosis, movilidad dentaria, sensibilidad a la percusión vertical u horizontal $(55,63-65)$. Las piezas dentarias deciduas no deben ser reimplantadas (61).

\section{CONSECUENCIAS \\ POSTERIORES AL \\ TRAUMATISMO \\ DENTOALVEOLAR EVIDENTES \\ IMAGENOLÓGICAMENTE}

Las complicaciones posteriores a traumatismos dentoalveolares pueden desarrollarse hasta meses o años después de la lesión. Con mayor frecuencia se presentan evidentes imagenológicamente: reabsorción radicular, obliteración del conducto radicular, anquilosis, periodontitis apical y, clínicamente, necrosis pulpar, cambio de color a nivel coronal $(5,6,12,15,66-68)$. Las lesiones traumáticas deben un tener registro previo con radiografía periapical estandarizada y radiografía panorámica para comparar con las otras piezas dentarias opuestas (69), por lo que en estas lesiones es importante realizar controles. Aumenta la posibilidad de reabsorción radicular interna o externa y la pérdida de soporte óseo la reposición quirúrgica de la pieza $(16,27)$. En la obliteración del conducto radicular se observa un estrechamiento progresivo del conducto radicular; deben realizarse controles con radiografías periapicales estandarizadas y prueba de vitalidad pulpar $(64,70)$. La periodontitis apical es el resultado de la infección bacteriana de la pulpa dental con su posterior diseminación hacia el ápice, lo que puede producir reabsorción radicular, que está relacionada con el daño al periodonto en el momento del trauma. Se observa imagenológicamente como imagen radio lúcida a nivel apical (21, 26, 67).

La característica radiográfica de la reabsorción radicular externa es el redondeo apical. Se pueden realizar controles con radiografía periapical; sin embargo, una radiografía panorámica calibrada 
(mismo técnico, misma máquina, mismo parámetro de posición) puede registrar la reabsorción con medición y comparación con su contralateral $(58,71,72)$. Para controlar el desarrollo apical de la pieza dentaria inmadura con reabsorción apical, es importante interrelacionar con el endodoncista para estimular la neoformación ósea y cierre apical mediante hidróxido de calcio y MTA y, luego, realizar controles con radiografías periapicales estandarizadas (73).

Casos de intrusión tienen un mayor impacto a nivel apical, su complicación posterior es la anquilosis y reabsorción radicular; sea interna o externa. Para su control, se deben realizar radiografías periapicales estandarizadas $(23,27,53$, 74). En la luxación extrusiva es rara la reabsorción radicular o anquilosis (29). La anquilosis es la fusión de la superficie radicular con el hueso alveolar que comprometen la integridad del hueso alveolar, se manifiesta por lesiones graves como avulsión o intrusión (75), y suele aparecer en la superficie vestibular de la raíz; por tanto, el empleo de radiografía periapical o panorámica no es confiable para determinar el diagnóstico en etapas iniciales. En etapas avanzadas, se observa la ausencia del espacio del ligamento periodontal, la radiografía periapical muestra los niveles óseos y la radiografía panorámica muestra la comparación de longitud radicular con las otras piezas. Clínicamente, un sonido metálico a la percusión vertical es perceptible. La presencia de la anquilosis puede demorar hasta año después de la lesión, para una mejor evaluación se debe aplicar la TCHC, lo que elimina la complicación de trabajo al profesional, por permitir la observación en reconstrucciones tridimensionales $(26,49,65,67,75)$. La anquilosis afecta la cresta alveolar, lo que detiene el desarrollo y causa el defecto óseo, lo que afecta el crecimiento facial continuo del niño, además de infraoclusión $(41,43$, 75).

\section{CONCLUSIONES}

La relevancia de la investigación del presente artículo es proporcionar información al profesional odontólogo sobre la importancia del manejo multidisciplinario de los traumatismos de los dientes, la evaluación clínica y el empleo necesario de la radiología, sea 2D o 3D, para establecer el diagnóstico y, posteriormente, el tratamiento con controles periódicos de la evaluación de las lesiones, ya que afectan al paciente en cualquier etapa de la vida. En la literatura, la información disponible de los traumatismos dentoalveolares y sus tejidos de soporte son reportes de casos clínicos aislados.

Contribución del autor: Mónica Pamela Sánchez Herrera ha participado en la concepción del artículo, la recolección de datos, su redacción y aprobación de la versión final.

Fuente de financiamiento: Autofinanciada.

Conflictos de interés: La autora declara no tener conflictos de interés de ningún tipo. 


\section{REFERENCIAS BIBLIOGRÁFICAS}

1. Das UM, Viswanath D, Subramanian V, Agarwal M. Management of dentoalveolar injuries in children: a case report. J Indian Soc Pedod Prev Dent. 2007; 25 (4): 183-6.

2. Kumaraswamy SV, Madan N, Keerthi R, Singh DS. Pediatric injuries in maxillofacial trauma: a 5 year study. J Maxillofac Oral Surg. 2009; 8 (2): 150-3.

3. Azami-Aghdash S, Ebadifard Azar F, Pournaghi Azar F, Rezapour A, Moradi-Joo $M$, Moosavi A, et al. Prevalence, etiology, and types of dental trauma in children and adolescents: systematic review and meta-analysis. Med J Islam Repub Iran. 2015; 29 (4): 234.

4. Pedrini D, Panzarini SR, Tiveron ARF, Abreu VM, Sonoda CK, Poi WR, et al. Evaluation of cases of concussion and subluxation in the permanent dentition: a retrospective study. J Appl Oral Sci. 2018; 26: e20170287.

5. Mahmoodi B, Rahimi-Nedjat R, Weusmann J, Azaripour A, Walter C, Willershausen $\mathrm{B}$. Traumatic dental injuries in a university hospital: a four-year retrospective study. BMC Oral Health. 2015; 15 (1): 139.

6. Moule A, Cohenca N. Emergency assessment and treatment planning for traumatic dental injuries. Aust Dent J. 2016; 61 Suppl 1: 21-38.

7. Braun TL, Xue AS, Maricevich RS. Differences in the management of pediatric facial trauma. Semin Plast Surg. 2017; 31 (2): 118-22.

8. Bernardino IM, Barbosa KGN, Nobrega LM, Cavalcante GMS, Ferreira EFE, d'Avila S. Interpersonal violence, circumstances of aggressions and patterns of maxillofacial injuries in the metropolitan area of Campina Grande, State of Paraiba, Brazil (2008-2011). Cien Saude Colet. 2017; 22 (9): 3033-44.

9. Lee CW, Foo QC, Wong LV, Leung YY. An overview of maxillofacial trauma in oral and maxillofacial tertiary Trauma Centre, Queen Elizabeth Hospital, Kota Kinabalu, Sabah. Craniomaxillofac Trauma Reconstr. 2017; 10 (1): 16-21.

10. Ferres-Amat E, Díaz-Martínez C, Herrera-Martínez S, Maura-Solivellas I, FerresPadro E. Unusual transalveolar and transmuco-gingival root avulsion of a fractured primary central incisor: a case with an 8-year follow-up. Case Rep Dent. 2015;2015:914846.

11. Singh G, Mohammad S, Pal US, Hariram, Malkunje LR, Singh N. Pediatric facial injuries: it's management. Natl J Maxillofac Surg. 2011; 2 (2): 156-62.

12. Pedrini D, Panzarini SR, Poi WR, Sundefeld ML, Tiveron AR. Dentists' level of knowledge of the treatment plans for periodontal ligament injuries after dentoalveolar trauma. Braz Oral Res. 2011; 25 (4): 307-13.

13. Maloney K. Circummandibular wires for treatment of dentoalveolar fractures adjacent to edentulous areas: a report of two cases. Craniomaxillofac Trauma Reconstr. 2015; 8 (3): 246-50. 
14. Iwase $M$, Ito $M$, Katayama $H$, Nishijima $H$, Shimotori $H$, Fukuoka $A$, et al. Traumatic displacement of maxillary permanent canine into the vestibule of the mouth. Case Rep Dent. 2015; 2015: 360160.

15. Andreasen JO, Lauridsen E, Gerds TA, Ahrensburg SS. Dental trauma guide: a source of evidence-based treatment guidelines for dental trauma. Dent Traumatol. 2012; 28 (5): 345-50.

16. Zaleckiene V, Peciuliene V, Brukiene V, Drukteinis S. Traumatic dental injuries: etiology, prevalence and possible outcomes. Stomatologija. 2014; 16 (1): 7-14.

17. Nagaveni NB, Umashankara KV. Tooth fragment embedded in the lower lip for 10 months following dentoalveolar trauma: A case report with literature review. Burns Trauma. 2014; 2 (3): 141-5.

18. Polat HB, Ay S, Kara MI. Maxillary tuberosity fracture associated with first molar extraction: a case report. Eur J Dent. 2007; 1 (4): 256-9.

19. Da Silva BR, Moreira Neto JJ, Da Silva FI Jr., De Aguiar AS. Finite element analysis applied to dentoalveolar trauma: methodology description. ISRN Dent. 2011; 2011: 297132.

20. Kulkarni VK, Sharma DS, Banda NR, Solanki M, Khandelwal V, Airen P. Clinical management of a complicated crown-root fracture using autogenous tooth fragment: A biological restorative approach. Contemp Clin Dent. 2013; 4 (1): 84-7.

21. Hecova $\mathrm{H}$, Tzigkounakis V, Merglova V, Netolicky J. A retrospective study of 889 injured permanent teeth. Dent Traumatol. 2010; 26 (6): 466-75.

22. Spinas E, Mameli A, Giannetti L. Traumatic Dental Injuries Resulting from Sports Activities; Immediate Treatment and Five Years Follow-Up: An Observational Study. Open Dent J. 2018; 12: 1-10.

23. C TSO, F MAdC, L COG, J MNdS, L FRG, A AFM, et al. Mineral Trioxide Aggregate for Intruded Teeth with Incomplete Apex Formation. Bull Tokyo Dent Coll. 2018; 59 (1): 35-41.

24. De Rossi M, De Rossi A, Queiroz AM, Nelson Filho P. Management of a complex dentoalveolar trauma: a case report. Braz Dent J. 2009; 20 (3): 259-62.

25. Faus-Matoses V, Martínez-Vinarta M, Alegre-Domingo T, Faus-Matoses I, FausLlacer VJ. Treatment of multiple traumatized anterior teeth associated with an alveolar bone fracture in a 20-year-old patient: A 3-year follow up. J Clin Exp Dent. 2014; 6 (4): e425-9.

26. Kindelan SA, Day PF, Kindelan JD, Spencer JR, Duggal MS. Dental trauma: an overview of its influence on the management of orthodontic treatment. Part 1. J Orthod. 2008; 35 (2): 68-78.

27. Jain V, Gupta R, Duggal R, Parkash H. Restoration of traumatized anterior teeth by interdisciplinary approach: report of three cases. J Indian Soc Pedod Prev Dent. 2005; 23 (4): 193-7. 
28. Murthy PS, Shivamallu AB, Deshmukh S, Nandlal B, Thotappa SK. Guided bone regeneration: A novel approach in the treatment of pediatric dentoalveolar trauma. Dent Res J (Isfahan). 2015; 12 (3): 285-8.

29. Serra-Pastor B, Penarrocha-Diago M, Penarrocha-Diago M, Agustin-Panadero R. Treatment and restoration of adult dentoalveolar trauma: A clinical case report. J Clin Exp Dent. 2016; 8 (5): e634-e7.

30. Imai T, Sukegawa S, Kanno T, Fujita G, Yamamoto N, Furuki Y, et al. Mandibular fracture patterns consistent with posterior maxillary fractures involving the posterior maxillary sinus, pterygoid plate or both: CT characteristics. Dentomaxillofac Radiol. 2014; 43 (2): 20130355.

31. Alkhadra T, Preshing W, El-Bialy T. Prevalence of traumatic dental injuries in patients attending University of Alberta Emergency Clinic. Open Dent J. 2016; 10: 315-21.

32. Turkaslan S, Turna $\mathrm{C}$. The esthetic rehabilitation of misplaced dental arch after fracture of anterior maxillae: a case report. Cases J. 2009; 2: 8723.

33. Samman M, Ahmed SW, Beshir H, Almohammadi T, Patil SR. Incidence and pattern of mandible fractures in the Madinah Region: A retrospective study. $J$ Nat Sci Biol Med. 2018; 9 (1): 59-64.

34. Rezende FM, Gaujac C, Rocha AC, Peres MP. A prospective study of dentoalveolar trauma at the Hospital das Clinicas, Sao Paulo University Medical School. Clinics (São Paulo). 2007; 62 (2): 133-8.

35. Fariniuk LF, Souza MH, Westphalen VP, Carneiro E, Silva Neto UX, Roskamp L, et al. Evaluation of care of dentoalveolar trauma. J Appl Oral Sci. 2010; 18 (4): 343-5.

36. Costa AL, Yasuda CL, Franca MC, Jr., Morita ME, Cendes F. Refractory epilepsy is highly associated with severe dentoalveolar and maxillofacial injuries. Epileptic Disord. 2011; 13 (1): 61-4.

37. Yuzugullu B, Polat O, Ungor M. Multidisciplinary approach to traumatized teeth: a case report. Dent Traumatol. 2008; 24 (5): e27-30.

38. Canoglu H, Gungor HC, Cehreli ZC. Management of cervical root fracture using orthodontic extrusion and crown reattachment: a case report. Oral Surg Oral Med Oral Pathol Oral Radiol Endod. 2007; 104 (3): e46-9.

39. C SK, Rao A, K S, G HR. Multidisciplinary Approach in Management of Fractured Central Incisor through Composite Plug Stabilization - A Case Report. J Int Oral Health. 2013; 5 (1): 79-82.

40. Franceschi RL, Drechsel L, Schuldt Filho G. Application of immediate dentoalveolar restoration in alveolus compromised with loss of immediate implant in esthetic area. Case Rep Dent. 2018; 2018: 1672170.

41. Steiner DR, West JD. Orthodontic-endodontic treatment planning of traumatized teeth. Semin Orthod. 1997; 3 (1): 39-44. 
42. Kang SH, Ha JH, Jin MU, Kim SK, Kim YK. Esthetic enhancement of a traumatized anterior tooth with a combination of forced eruption and tooth alignment: a case report. Restor Dent Endod. 2016; 41 (3): 210-7.

43. Tsurumachi T, Kuno T. Autotransplantation of a maxillary first premolar to replace an ankylosed maxillary incisor: 7-year follow-up. Int Endod J. 2011; 44 (9): 863-75.

44. Anand PS, Ashok S, Nandakumar K, Varghese NO, Kamath KP. Surgical exposure and crown lengthening for management of complicated fractures of maxillary anterior teeth. A case report. NY State Dent J. 2013; 79 (6): 41-6.

45. Heda CB, Heda AA, Kulkarni SS. A multi-disciplinary approach in the management of a traumatized tooth with complicated crown-root fracture: A case report. $J$ Indian Soc Pedod Prev Dent. 2006; 24 (4): 197-200.

46. Tondelli PM, Mendonca MR, Cuoghi OA, Pereira AL, Busato MC. Knowledge on dental trauma and orthodontic tooth movement held by a group of orthodontists. Braz Oral Res. 2010; 24 (1): 76-82.

47. Zaleckiene V, Peciuliene V, Brukiene V, Jakaitiene A, Aleksejuniene J, Zaleckas L. Knowledge about traumatic dental injuries in the permanent dentition: A survey of Lithuanian dentists. Dent Traumatol. 2018; 34 (2): 100-6.

48. Park W, Lee HN, Jeong JS, Kwon JH, Lee GH, Kim KD. Optimal protocol for teleconsultation with a cellular phone for dentoalveolar trauma: an in-vitro study. Imaging Sci Dent. 2012; 42 (2): 71-5.

49. Ahmad M, Jenny J, Downie M. Application of cone beam computed tomography in oral and maxillofacial surgery. Aust Dent J. 2012; 57 Suppl 1: 82-94.

50. Spurrier SW, Hall SH, Joondeph DR, Shapiro PA, Riedel RA. A comparison of apical root resorption during orthodontic treatment in endodontically treated and vital teeth. Am J Orthod Dentofacial Orthop. 1990; 97 (2): 130-4.

51. Hogg NJ, Horswell BB. Hard tissue pediatric facial trauma: a review. J Can Dent Assoc. 2006; 72 (6): 555-8.

52. Guven Y, Zorlu S, Cankaya AB, Aktoren O, Gencay K. A Complex facial trauma case with multiple mandibular fractures and dentoalveolar injuries. Case Rep Dent. 2015; 2015: 301013.

53. Bauss O, Schafer W, Sadat-Khonsari R, Knosel M. Influence of orthodontic extrusion on pulpal vitality of traumatized maxillary incisors. J Endod. 2010; 36 (2): 203-7.

54. Mendes L, Laxe L, Passos L. Ten-year follow-up of a fragment reattachment to an anterior tooth: a conservative approach. Case Rep Dent. 2017; 2017: 2106245.

55. Saoud TM, Mistry S, Kahler B, Sigurdsson A, Lin LM. Regenerative endodontic procedures for traumatized teeth after horizontal root fracture, avulsion, and perforating root resorption. J Endod. 2016; 42 (10): 1476-82.

56. Tzanetakis GN. Management of Intruded Immature Maxillary Central Incisor with Pulp Necrosis and Severe External Resorption by Regenerative Approach. J Endod. 2018; 44 (2): 245-9. 
57. Medeiros RB, Mucha JN. Immediate vs late orthodontic extrusion of traumatically intruded teeth. Dent Traumatol. 2009; 25 (4): 380-5.

58. Cehreli ZC, Sara S, Aksoy B. Revascularization of immature permanent incisors after severe extrusive luxation injury. J Can Dent Assoc. 2012; 78: c4.

59. Gorur I, Orhan K, Can-Karabulut DC, Orhan AI, Ozturk A. Low-level laser therapy effects in traumatized permanent teeth with extrusive luxation in an orthodontic patient. Angle Orthod. 2010; 80 (5): 968-74.

60. Navit S, Shahi N, Khan SA, Sharma A, Singh V, Mishra RP, et al. Nature's benefaction as a life saver for an avulsed tooth: an in vitro study. J Clin Diagn Res. 2017; 11 (6): ZC01-ZC4.

61. Ceallaigh PO, Ekanaykaee K, Beirne CJ, Patton DW. Diagnosis and management of common maxillofacial injuries in the emergency department. Part 5: Dentoalveolar injuries. Emerg Med J. 2007; 24 (6): 429-30.

62. Zhu W, Zhang Q, Zhang Y, Cen L, Wang J. PDL regeneration via cell homing in delayed replantation of avulsed teeth. J Transl Med. 2015; 13: 357.

63. Closs LQ, Reston EG, Vargas IA, de Figueiredo JA. Orthodontic space closure of lost traumatized anterior teeth - case report. Dent Traumatol. 2008; 24 (6): 687-90.

64. Heithersay GS. Life cycles of traumatized teeth: long-term observations from a cohort of dental trauma victims. Aust Dent J. 2016; 61 Suppl 1: 120-7.

65. Bendoraitiene E, Zemgulyte S, Borisovaite M. Reasonable outcome of avulsed permanent upper incisor after seven years follow-up period: a case report. J Oral Maxillofac Res. 2017; 8 (4): e6.

66. DiBiase AT, Woodhouse NR, Papageorgiou SN, Johnson N, Slipper C, Grant J, et al. Effect of supplemental vibrational force on orthodontically induced inflammatory root resorption: A multicenter randomized clinical trial. Am J Orthod Dentofacial Orthop. 2016; 150 (6): 918-27.

67. Yamashita FC, Previdelli ITS, Pavan NNO, Endo MS. Retrospective study on sequelae in traumatized permanent teeth. Eur J Dent. 2017; 11 (3): 275-80.

68. Ebrahim FH, Kulkarni G. Fixed orthodontic appliances in the management of severe dental trauma in mixed dentition: a case report. J Can Dent Assoc. 2013; 79: d131.

69. Bauss O, Rohling J, Sadat-Khonsari R, Kiliaridis S. Influence of orthodontic intrusion on pulpal vitality of previously traumatized maxillary permanent incisors. Am J Orthod Dentofacial Orthop. 2008; 134 (1): 12-7.

70. Bauss O, Rohling J, Rahman A, Kiliaridis S. The effect of pulp obliteration on pulpal vitality of orthodontically intruded traumatized teeth. J Endod. 2008; 34 (4): 417-20.

71. Llamas-Carreras JM, Amarilla A, Espinar-Escalona E, Castellanos-Cosano L, Martín-González J, Sánchez-Domínguez B, et al. External apical root resorption in maxillary root-filled incisors after orthodontic treatment: a split-mouth design study. Med Oral Patol Oral Cir Bucal. 2012; 17 (3): e523-7. 
72. Nazzal H, Kenny K, Altimimi A, Kang J, Duggal MS. A prospective clinical study of regenerative endodontic treatment of traumatized immature teeth with necrotic pulps using bi-antibiotic paste. Int Endod J. 2018; 51 Suppl 3: e204-e15.

73. Guzeler I, Uysal S, Cehreli ZC. Management of trauma-induced inflammatory root resorption using mineral trioxide aggregate obturation: two-year follow up. Dent Traumatol. 2010; 26 (6): 501-4.

74. Hamilton RS, Gutmann JL. Endodontic-orthodontic relationships: a review of integrated treatment planning challenges. Int Endod J. 1999; 32 (5): 343-60.

75. Chang HY, Chang YL, Chen HL. Treatment of a severely ankylosed central incisor and a missing lateral incisor by distraction osteogenesis and orthodontic treatment. Am J Orthod Dentofacial Orthop. 2010; 138 (6): 829-38.

Correspondencia: Mónica Pamela Sánchez Herrera, p.sanchez.herrera04@ gmail.com ARtí́culo RECIBIDO: 07/08/2018

Artículo aCEPTAdo: 29/10/2018 